\title{
A Comparison of Papoulis and Chebyshev Filters in the Continuous Time Domain
}

\author{
Negovan STAMENKOVIC ${ }^{1}$, Nikola STOJANOVIC ${ }^{2}$, Dijana JOVANOVIC ${ }^{3}$, Zeljko STANKOVIC ${ }^{4}$ \\ ${ }^{1}$ University of Priština, Faculty of Sciences, 38220 K. Mitrovica, Serbia \\ ${ }^{2}$ Faculty of Electronic Engineering, University of Niš, A. Medvedeva 14, 18106 Niš, Serbia \\ ${ }^{3}$ Department of Informatics, College of academic studies "Dositej", 11000 Belgrade \\ ${ }^{4}$ Faculty of Informatics, Paneuropean University Apeiron, 78000 Banja Luka, Bosnia and Herzegovina
}

negovan.stamenkovic@pr.ac.rs, nikola.stojanovic@elfak.ni.ac.rs

Submitted March 21, 2021 / Accepted June 13, 2021

\begin{abstract}
The subject of this paper is the revisit of the Chebyshev (equiripple) and Papoulis (monotonic or staircase) low-pass filter in order to compare. It can be stated the fair comparison of Papoulis and Chebyshev filters cannot be found in the available literature. At the beginning, it is shown that ripple parameter may be used in order the Chebyshev filter to obtain a magnitude response having less passband ripple than the standard Chebyshev response. At the same time, the passband edge frequency is preserved at $3 \mathrm{~dB}$. Further, the unified approach to design odd and even degree Papoulis filters is explained. For the purpose of comparison, the Chebyshev filter as a counterpart of the Papoulis filter is introduced. Thus obtained Chebyshev filter has the same stop band insertion loss, group delay and transient response as Papoulis filter. However, its passband performance is much better. It is shown that Chebyshev filter counterpart offers a better solution than Papoulis filter in all applications, except in ones applications where is required that passband attenuation to have a staircase shape.
\end{abstract}

\section{Keywords}

Electronic filters, approximation, Chebyshev filter, Papoulis filter, insertion loss, return loss

\section{Introduction}

The Papoulis propose allpole lowpass filters [1] which may be considered as a good transition between Butterworth filters from one side and Chebyshev filters on the other. The passband amplitude characteristic of these filters decreases monotonically with $\omega$ and exhibits staircase behavior. "These filters can be used in many applications; i.e., when the transient response is also considered, a high ripple in the passband is not tolerated; one then uses as a simple compromise the Butterworth filter whose cutoff properties are not too good.", considers the author of the mentioned paper. After the publication of that paper, the monotony filters have attracted the attention of researchers. For examples, they are: the class $\mathrm{H}$ (Halpern) filters [2] which yield maximum asymptotic slope, the monotonic filters with improved stopband performance [3], LSM (least-square monotonic) filters [4] that provide the smallest passband loss among all filters whose passband magnitude response is bound to be monotonic and paper [5] in which authors shown that the Halpern filters are only of academic interest. Comparison of monotonic and parabolic filter is given in [6], while in paper [7] comparison among monotonic filters can be found. It should be also noted that monotonous filters occupy a large part of the recently published book [8].

This paper has two main objectives. Firstly, it will be shown that equiripple (Chebyshev) approximation offers better or in the worst case equal performances in comparison with staircase (Papoulis) approximation. Secondly, it has been shown that Chebyshev filter, as a counterpart of Papoulis filter, can be used instead of the Papoulis filter in all applications. Finally, we got the general feeling that staircase filters are only of academic importance.

\section{Transfer Function of the Filters}

Assume that a linear time-invariant doubly terminated network is described by a linear differential equation of degree $n$. The corresponding power loss [9] is a rational function of $s$ and takes the form:

$$
\left|t_{n}^{2}(\mathrm{j} \omega)\right|=\frac{1}{1+\phi_{n}\left(\omega^{2}\right)}
$$

with $\phi_{n}\left(\omega^{2}\right)$ being the characteristic function complete even or odd polynomial of degree $n$ in $\omega^{2}$. Insertion loss expressed in $\mathrm{dB}$ is given by $I_{\mathrm{L}}=20 \log _{10}\left|t_{n}(\mathrm{j} \omega)\right|$.

The relationship between the power absorbed by the load resistor and the power which reflected at input terminals back to the source is given by the Feldtkeller equation $\left|t_{n}(\mathrm{j} \omega)\right|^{2}+\left|\rho_{n}(\mathrm{j} \omega)\right|^{2}=1$, where $\rho(\mathrm{j} \omega)$ is reflection coefficient at input ports. Putting expression for transmission 
coefficient (1) into the Feldtkeller's equation, one gets

$$
\left|\rho_{n}(\mathrm{j} \omega)\right|^{2}=\frac{\phi_{n}\left(\omega^{2}, \varepsilon\right)}{1+\phi_{n}\left(\omega^{2}, \varepsilon\right)}
$$

and return loss expressed in $\mathrm{dB}$ is given in the following form $R_{\mathrm{L}}=20 \log _{10}\left|\rho_{n}(\mathrm{j} \omega)\right|$.

Having established $\phi_{n}\left(\omega^{2}\right)$ the continuous time lowpass transfer functions $t_{n}(s)$ can be found by using the standard procedure. The first step is the analytic continuation in the complex $s$-plane ${ }^{1}$ of $\phi_{n}\left(\omega^{2}\right)$ by substitution $\omega^{2} \rightarrow-s^{2}$. The second step is factoring $1+\phi_{n}\left(-s^{2}\right)$. The left half of the $s$-plane factors being the desired denominators of stable and time invariant transfer function:

$$
t_{n}(s)=\frac{h_{0}}{\prod_{i=1}^{n}\left(s-s_{i}\right)}=\frac{h_{0}}{\sum_{i=1}^{n+1} a_{i} s^{n-i+1}}=\frac{1}{\sum_{i=1}^{n+1} d_{i} s^{n-i+1}}
$$

where $h_{0}=a_{n+1} / \sqrt{1+\phi_{n}^{2}(0)}$ is constant that ensures that amplitude $\left|t_{n}(\omega)\right|$ is bounded above by unity, and $d_{i}=a_{i} / h_{0}$. If $n$ is odd then the characteristic function is equal to zero at the zero frequency, i.e. $t_{n}(0)=1$, which gives $h_{0}=a_{n+1}$ and $d_{n+1}=1$.

\subsection{Scaled Chebyshev Characteristic Function}

As well known, the optimum filter design with respect to the cutoff slope can be obtained using Chebyshev polynomials [10]. When a high ripple in the filter's pass-band cannot be tolerated, to reduce the pass-band ripples and to preserve the pass-band edge frequency at $\omega_{3 \mathrm{~dB}}=1$ is necessary. Therefore the scaling the standard Chebyshev transcendental function with the ripple parameter $\varepsilon$ and the renormalizing with respect to the real angular frequency $\xi_{n}(\varepsilon)$ will be used. The scaled Chebtshev characteristic function of the lowpass filter can be now obtained in the following form

$$
\phi_{n}\left(\omega^{2}, \varepsilon\right)=\varepsilon^{2} \cos ^{2}\left(n \arccos \left(\omega \cdot \xi_{n}(\varepsilon)\right)\right) .
$$

Since the $3 \mathrm{~dB}$ passband cutoff frequency normalized to unity is considered, i.e. $\phi_{n}(1, \varepsilon)=1$, the angular frequency for renormalization of the characteristic function can easily be found in the closed form as follows

$$
\xi_{n}(\varepsilon)=\cos \left(\frac{1}{n} \arccos \left(\frac{1}{\varepsilon}\right)\right) \geq 1 .
$$

Thus, on the interval $\left[0, \omega_{\mathrm{rb}}\right]$, where $\omega_{\mathrm{rb}}=1 / \xi_{n}(\varepsilon) \leq 1$ is the ripple band, $\phi_{n}\left(\omega^{2}, \varepsilon\right)$ oscillates between 0 and $\varepsilon^{2}$, causing the $\left|t_{n}(\mathrm{j} \omega, \varepsilon)\right|$ to oscillate between 1 and $1 / \sqrt{1+\varepsilon^{2}}$, while at the same time $\left|t_{n}(\mathrm{j}, \varepsilon)\right|=1 / \sqrt{2}$. Insertion loss level over the ripple band can be computed as $L_{\mathrm{r}}=10 \log _{10}\left(1+\varepsilon^{2}\right)$.

It can be noted that characteristic function (4) is squared Chebyshev polynomial of the degree $n$, scaled with $\varepsilon$ and renormalised by $\omega_{\mathrm{rb}}=1 / \xi_{n}(\varepsilon)$, which has the form

$$
\mathcal{T}_{n}^{(\varepsilon)}(\omega)=\varepsilon \cos \left[n \arccos \left(\frac{\omega}{\omega_{\mathrm{rb}}}\right)\right] .
$$

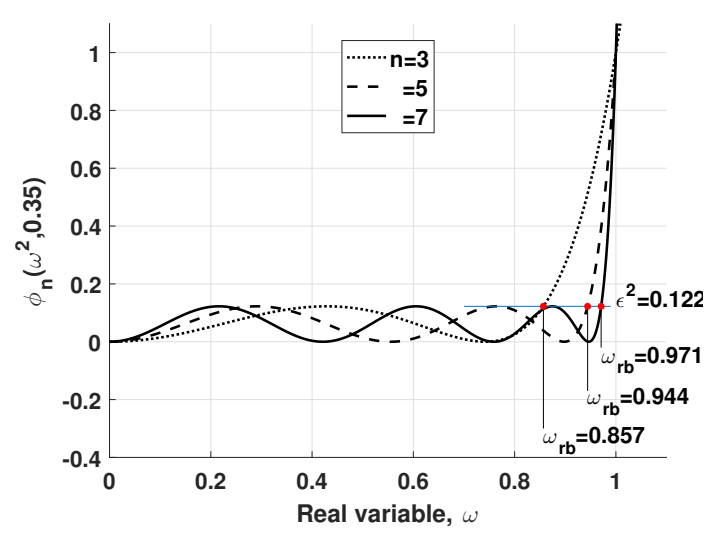

Fig. 1. Scaled Chebyshev characteristic functions for $\varepsilon=0.35$ and $n=3,5$ and 7. Ripple bands, $\omega_{\mathrm{rb}}$, are labeled.

Hereinafter it is referred to as Scaled Chebyshev polynomial or only Chebyshev polynomial since his pass band is equiripple. It can be also considered that ripple factor $\varepsilon$ can be referred as the order of the Scaled Chebyshev polynomial. Embedded parameter $\varepsilon$ act as a degree of freedom in the same away as order $v$ in the case of Gegenbauer polynomials [11]. These polynomials are not orthogonal with respect to the Chebyshev weighting function $\left(1-\omega^{2}\right)^{-0.5}$ over the interval $\omega \in[-1,1]$, since the renormalized frequency $\omega_{\mathrm{rb}}$ depends both on the filter's degree and the ripple parameter $\varepsilon$. On the other hand, these polynomials are pure even or pure odd polynomials with real roots lying in the passband.

The plot of three Scaled Chebyshev characteristic functions, $\phi_{n}(\omega, \varepsilon)$, for $\varepsilon=0.35$ and $n=3,5$ and 7 are given in Fig. 1.

Ripple bands $\left[0, \omega_{\mathrm{rb}}\right]$ of these characteristic functions are also depicted in Fig. 1. Thus, the Scaled Chebyshev polynomials (6) are the variety of Chebyshev polynomials and they can be used in the approximation of the filter magnitude function of the transmission coefficient.

\subsection{Characteristic Function of Papoulis Filter}

In the original papers [1] and [12] Professor Papoulis proposed monotonic (staircase) lowpass filters for the odd and even degree ${ }^{2}$, respectively. The class of filters is called L-filters, because in the original derivation Legendre polynomials were used. In the following text a unique solution for the odd and even a filter degree in details is described.

The generating equation for the odd and even characteristic function of the staircase case filter of the degree $n$ with real coefficients, can be expressed in the following form [14]

$$
\phi_{n}\left(\omega^{2}\right)=\int_{0}^{\omega} x u_{n-1}^{2}\left(x^{2}\right) \mathrm{d} x
$$

and it is positive real function of $\omega^{2}$. To achieve that $\phi_{n}\left(\omega^{2}\right)$ is a monotonic polynomial $\left(\mathrm{d} \phi_{n}\left(\omega^{2}\right) / \mathrm{d} \omega \geq 0\right)$, the polynomial $u_{n-1}^{2}\left(x^{2}\right)$ is used to be a perfect square. To determine $u_{n-1}\left(x^{2}\right)$, it is expanded as a sum of squared orthogonal

\footnotetext{
${ }^{1} s=\sigma+\mathrm{j} \omega$

2 The same results for even degree were published by Fukada [13].
} 
polynomials in the interval of orthogonality matching the normalized passband of the lowpass filter, i.e. $\omega \in[0,1]$. Using shifted Jacobi's polynomials instead of Legendre polynomials [1] the staircase (monotonic) Papoulis filter can also be derived.

The Jacobi polynomial of degree $n$ with two inherent parameters $\alpha$ and $\beta, P_{n}^{(\alpha, \beta)}(x)$, is orthogonal with respect to the weight function $w(x)=(1-x)^{\alpha}(1+x)^{\beta}$ over the interval $x \in[-1,1]$. It have $n$ distinct zeros for $\alpha \neq \beta$ but they are neither even nor odd. Such type of the polynomial is not suitable to be a filter characteristic function, and it needs to be modified in order to meet the requirements to be a filter function [15]. For the purpose of the monotonic filter design shifted Jacobi polynomials are used. They are defined by linear substitution, $G_{n}(p, q, y)=P_{n}^{(\alpha, \beta)}(2 y-1)$, where $p=\alpha+\beta+1$ and $q=\beta+1$ (with $q \geq 0$ and $p \geq q-1$ ). These polynomials are orthogonal on the interval $y \in[0,1]$ with respect to the weight function $w(y)=(1-y)^{p-q} y^{q-1}$. It can be shown that if $q \geq 1$ and $p=q$, then $w(y)=y^{q-1}$ and $G_{i}(q, q, 1)=1$. Shifted Jacobi polynomials can be easily determined through the Matlab symbolic function jacobiP $(n, a, b, x)$.

Since parameter values $p=q \leq 2$ are restricted to be the integer values and let $y=x^{2}$, then $G_{i}\left(q, q, x^{2}\right)$ is a pure even orthogonal polynomial with respect to the weight function $w(x)=x^{2 q-1}$. In other word, polynomial $u_{n-1}\left(x^{2}\right)=x^{q-1} G_{i}\left(q, q, x^{2}\right)$ is orthogonal wit respect to the weight $x$. Then

$$
2 \int_{0}^{1} x\left[x^{q-1} G_{n}\left(q, q, x^{2}\right)\right]\left[x^{q-1} G_{m}\left(q, q, x^{2}\right)\right] \mathrm{d} x=0
$$

with $\mathrm{d} y=2 x \mathrm{~d} x$ and it can be used in the certain place in the filter characteristic function.

In order to determine the characteristic function of the Papoulis filters (7), we first expand polynomial $u_{n-1}\left(x^{2}\right)$ into the form of a series of the shifted Jacobi orthonormal polynomials, the following expression arises

$$
\phi_{n}\left(q, \omega^{2}\right)=\int_{0}^{\omega} x\left[x^{q-1} \sum_{j=0}^{(n-q) / 2} c_{j} K_{j} G_{j}\left(q, q, x^{2}\right)\right]^{2} \mathrm{~d} x
$$

where $q$ is 2 for $n$ even and 1 for $n$ odd. The constant $K_{i}$ is calculated from the orthonormality condition for the polynomial $u_{n-1}\left(x^{2}\right)$. The inner product is first calculated for that purpose

$$
\int_{0}^{1} x\left[x^{q-1} G_{i}\left(q, q, x^{2}\right)\right]^{2} \mathrm{~d} x= \begin{cases}\frac{1}{4 i+2} & \text { for } i \text { odd } \\ \frac{1}{4 i+4} & \text { for } i \text { even }\end{cases}
$$

So, equation (10) implies $K_{i}=\sqrt{4 i+4}$, where the values for $i$ are bounded by $q / 2-1 \leq i \leq n / 2-1$ for $n$ even and $K_{i}=\sqrt{4 i+2}$, where the values for $i$ are bounded by $(q-1) / 2 \leq i \leq(n-1) / 2$ for $n$ odd. Since $\phi_{n}(q, 1)=1$ it follows that $\sum_{i} c_{i}^{2}=1$, then the $\omega_{3 \mathrm{~dB}}$ bandwidth is normalized to be unity.
From equation (9), the cutoff rate of the characteristic function at the passband edge frequency $\omega=1$

$$
\left.\frac{\mathrm{d} \phi_{n}\left(q, \omega^{2}\right)}{\mathrm{d} \omega}\right|_{\omega=1}=\left[\sum_{j=0}^{(n-q) / 2} c_{j} K_{j}\right]^{2}
$$

is maximum, as it is introduced by Papoulis [1]. The unknown coefficients $c_{j}$ can be determined by solving an extremal-value problem with the help of the Lagrange multiplier $\lambda$ and the Lagrange function as

$$
F(\mathbf{c}, \lambda)=\sum_{j=0}^{(n-q) / 2} c_{j} K_{j}-\lambda\left[\sum_{j=0}^{(n-q) / 2} c_{j}^{2}-1\right]=0
$$

where $\mathbf{c}$ is the vector contains coefficients $c_{j}$. The partial derivatives of $F(\mathbf{c}, \lambda)$ give $(n-q) / 2+1$ equations, which are solved by setting them to zero, as

$$
\frac{\partial F}{\partial c_{j}}=K_{i}-2 \lambda c_{j}=0, \quad \text { for } j=0,1, \ldots, \frac{n-q}{2}
$$

and

$$
\frac{\partial F}{\partial \lambda}=\sum_{j} c_{j}^{2}-1=0
$$

By using equation (13), the coefficients $c_{i}$ can be expressed as $c_{j}=K_{j} / 2 \lambda$. Putting these values into equation (14) parameter $\lambda$ is obtained and then $c_{i}$. Since the coefficients $c_{i}$ are known, the characteristic function is obtained by using definite integral as it is given in (9). For example, let $n=6$ and $q=2$. Then $i=0,1,2, \mathbf{c}=[\sqrt{6} / 6, \sqrt{3} / 3, \sqrt{2} / 2]$ and $\phi_{6}\left(2, \omega^{2}\right)=50 \omega^{12}-120 \omega^{10}+105 \omega^{8}-40 \omega^{6}+6 \omega^{4}$.

The plot of three odd degree Papoulis (staircase) characteristic functions $(q=1)$ for $n=3,5$ and 7 are given in Fig. 2. The inflection points closest to the passband edge are labeled. These frequencies are used to define the staircase band $\omega_{\mathrm{s}}$ and staircase insertion loss level, $L_{\mathrm{s}}$. If filter degree increases, the staircase passband increase and the correspond passband attenuation slightly increased.

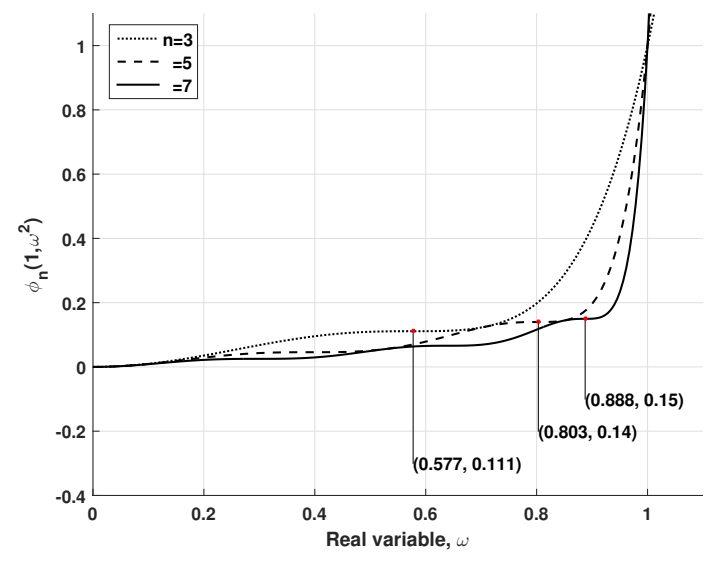

Fig. 2. Papoulis characteristic functions for $n=3,5$ and 7 . 


\begin{tabular}{|c|c|c|c|c|c|c|c|c|c|}
\hline \multicolumn{9}{|c|}{$D(s)=d_{1} s^{n}+d_{2} s^{n-1}+\cdots+d_{n} x+d_{n+1}$} & \multirow[b]{2}{*}{11} \\
\hline$n$ & 3 & 4 & 5 & 6 & 7 & 8 & 9 & 10 & \\
\hline$a_{1}$ & 1.7320508 & 2.4494898 & 4.4721360 & 7.07106782 & 13.2287569 & 22.1359444 & 42.0 & 72.7461319 & 139.298248 \\
\hline$a_{2}$ & 2.3079948 & 3.8345675 & 6.9871788 & 12.1278400 & 22.7857418 & 40.6406403 & 77.5139389 & 140.83223 & 271.064789 \\
\hline$a_{3}$ & 2.2697771 & 4.5777082 & 9.7567472 & 18.9272881 & 39.3909187 & 75.9192657 & 156.357117 & 301.549255 & 617.50238 \\
\hline$a_{4}$ & 1.0 & 2.9713612 & 7.4948750 & 16.9495487 & 38.3167419 & 81.1383209 & 176.202393 & 363.348633 & 773.079041 \\
\hline$a_{5}$ & & 1.0080065 & 3.9588523 & 11.4509706 & 30.5599766 & 73.5000687 & 176.926605 & 397.415161 & 911.598999 \\
\hline$a_{6}$ & & & 1.0 & 4.7394233 & 16.1055908 & 46.2091255 & 124.912025 & 311.501251 & 769.243103 \\
\hline$a_{7}$ & & & & 1.0065688 & 5.7465262 & 21.8450184 & 71.0707932 & 204.038589 & 562.743774 \\
\hline$a_{8}$ & & & & & 1.0 & 6.5706539 & 28.300066 & 98.7220383 & 311.780029 \\
\hline$a_{9}$ & & & & & & 1.0049937 & 7.58414555 & 35.8750725 & 138.497711 \\
\hline$a_{10}$ & & & & & & & 1.0 & 8.43657398 & 44.1699791 \\
\hline$a_{11}$ & & & & & & & & 1.00385964 & 9.45252609 \\
\hline$a_{12}$ & & & & & & & & & 1.0 \\
\hline$\varepsilon_{n}$ & 0.1831807 & 0.1267956 & 0.144913 & 0.1148077 & 0.118908 & 0.1000614 & 0.1009258 & 0.08794392 & 0.08781151 \\
\hline Area & 0.100025 & 0.0674987 & 0.0485481 & 0.0365567 & 0.0292278 & 0.0234262 & 0.0197498 & 0.0164727 & 0.0143424 \\
\hline AS & 1.7321 & 2.4495 & 4.4721 & 7.0711 & 13.2288 & 22.1359 & 42 & 72.7461 & 139.2982 \\
\hline$\omega_{\mathrm{rb}}$ & 0.7507 & 0.8022 & 0.8769 & 0.8966 & 0.9241 & 0.9339 & 0.9474 & 0.9532 & 0.9610 \\
\hline$L_{\mathrm{r}}[\mathrm{dB}]$ & 0.1433 & 0.0693 & 0.0903 & 0.0569 & 0.0610 & 0.0433 & 0.0440 & 0.0335 & 0.0334 \\
\hline
\end{tabular}

Tab. 1. The normalized coefficients of the polynomials in the denominator of the equiripple filters designed as the counterparts of staircase filters.

\section{The Chebyshev Filter as a Counter- part of the Papoulis Filter}

The first border class of the Scaled C filters, obtained when $\varepsilon \rightarrow 0$, correspond to the Butterworth filters. The second border class $\left(\varepsilon=1\right.$ and $\left.\xi_{n}(1)=1\right)$ corresponds to the Chebyshev filter with $3 \mathrm{~dB}$ passband ripples. One can observe, if the ripple parameter $\varepsilon$ increases from 0 to 1 , the asymptotic slope ${ }^{3}$ increase from 1 for Butterworth filters, to $2^{n-1}$ for Chebyshev filters, where $n$ is filter degree. The asymptotic slope of the Papoulis filter lies between these two. It will be shown in the following text that the Chebyshev filter can be considered as the counterpart of Papoulis filter, if it has the same asymptotic rate as Papoulis filter.

Since both filters should have the same asymptotic slope, the order $\varepsilon$ of the Chebyshev filter can be found by solving the following nonlinear equation $A S_{\mathrm{C}}=A S_{\mathrm{P}}$, where $A S_{\mathrm{C}}$ is asymptotic slope of Chebyshev filter

$$
A S_{\mathrm{C}}=\left[\frac{\mathrm{d}^{2 n}}{\mathrm{~d} \omega^{2 n}} \phi_{n}\left(\omega^{2}, \varepsilon\right)\right]^{\frac{1}{2}}=\varepsilon 2^{n-1} \cos ^{n}\left(\frac{1}{n} \arccos \frac{1}{\varepsilon}\right)
$$

and $A S_{\mathrm{P}}$ is known asymptotic slope of Papoulis filter. In other word, asymptotic slope is squared root of the leading coefficient of filter characteristic function $\phi_{n}\left(\omega^{2}\right)$. For example, if $n=9$ then asymptotic slope of Papoulis filter is $A S_{\mathrm{P}}=42$ and Chebyshev filter, as the counterpart of the Papoylis filter filter, is characterized by $\varepsilon_{9}=0.1009258$.

Table 1 contains coefficients of the Chebyshev filters as the counterparts of the Papoulis filters up to 11th degree. Table also summarizes the corresponding performances: the counterparts values of $\varepsilon_{n}$, the area under the characteristic function in the passband 4 (Area), the asymptotic slope (AS), the ripple band $\left(\omega_{\mathrm{rb}}\right)$, and insertion loss level over the ripple band $\left(L_{\mathrm{r}}\right)$. The insertion loss level of the Chebyshev filters (counterpart of Papoulis filter) is less than $L_{\mathrm{r}}<0.1 \mathrm{~dB}$ and decease if degree of filter increase. Therefore, it can be said that it is approximately monotonous in the passband [16].

Performances of Papoulise filter can be calculated by using the procedure proposed in Sec. 2.2, or there may be found in [7].

\section{Comparison}

The frequency responses of the 9th degree Papoulis, Chebyshev and Butterwort filters are given in Fig. 3. Reducing order $\varepsilon$ of the Chebyshev filter to the value $\varepsilon_{9}=$ 0.1009258 makes the group delay response and the stop band insertion-loss of the Chebyshev filter the same as is those of the Papoulis filter. Both filters have the same asymptotic slope, as can be seen in Fig. 3.

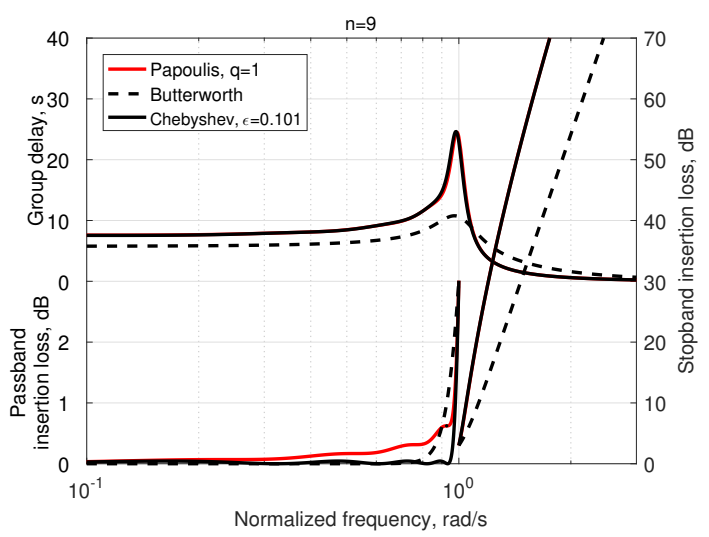

Fig. 3. Frequency responses of Papoulis filter and Chebyshev filter as its counterpart.

\footnotetext{
${ }^{3}$ The asymptotic slope will be expressed as the square root of the leading coefficient in characteristic function $\phi_{n}\left(\omega^{2}\right)$. Asymptotic slope of a Chebyshev filter with $3 \mathrm{~dB}$ passband ripple is $A S_{\mathrm{C}}=2^{n-1}$.

${ }^{4}$ The area required is obtained by integration: Area $=\int_{0}^{1} \phi\left(\omega^{2}, \varepsilon\right) \mathrm{d} \omega$, and it is in the relation to reflection power at the input terminals of the filter.
} 


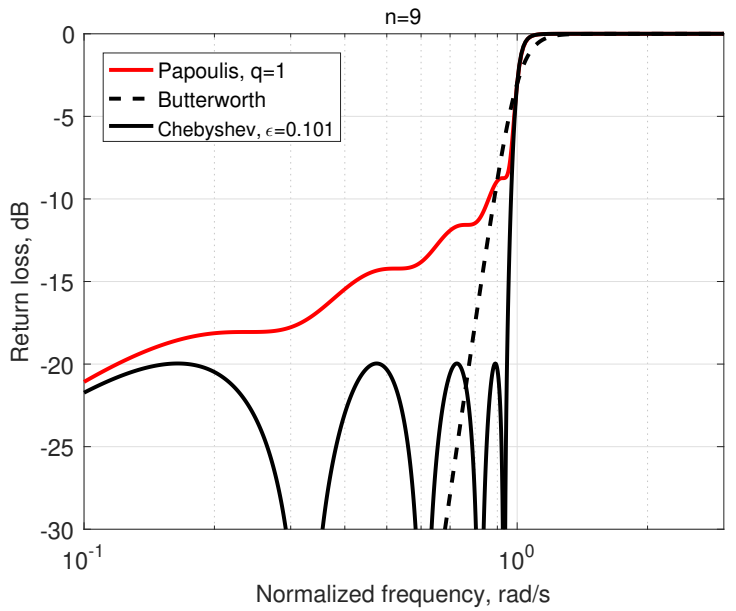

Fig. 4. Return losses of Papoulis filter and Chebyshev filter as its counterpart.

Chebyshev filters offer better performances in the passband. Insertion loss level over ripple band, $L_{\mathrm{r}}=0.044 \mathrm{~dB}$, is much lower than the insertion loss level over staircase band, $L_{\mathrm{s}}=0.6229 \mathrm{~dB}$. The staircase band $\omega_{\mathrm{s}}=0.9275$ is slightly narrower than ripple band $\omega_{\mathrm{rp}}=0.9474$. The area under the staircase characteristic function Area $_{\mathrm{s}}=0.0639$ is almost four times larger than the area under equiripple characteristic function Area $=0.01975$. In other words, the reflection power of Papoulis filter is about four times larger.

The return loss responses of the filters whose frequency responses are given in Fig 3, can be seen in Fig. 4. The return loss level of the Chebyshev filter is $R_{\mathrm{L}}=-19.9640 \mathrm{~dB}$ and it is about $12 \mathrm{~dB}$ under the return loss level of the Papoulis filter.

The zeroes of the reflection coefficient can be obtained by finding the roots of the Scaled C polynomial (6). Solving $\mathcal{T}_{n}^{(\varepsilon)}(-\mathrm{j} s)=0$, yields the reflection zeros in the closed form

$$
s_{k}=\mathrm{j} \frac{\cos \left((2 k+1) \frac{\pi}{2 n}\right)}{\cos \left(\frac{1}{n} \arccos \frac{1}{\varepsilon}\right)}, \quad \text { for } k=0,1, \ldots,\left\lfloor\frac{n-1}{2}\right\rfloor
$$

where $\lfloor x\rfloor$ denotes the flooring function. Obviously, all reflection zeros lie on the $\mathrm{j} \omega$-axis in the passband, and maximum power transmission through the filter occurs at these frequencies.

There are no differences between unite step responses of Papoulis and Chebyshev filter as its counterpart, as is shown in Fig. 5.

As explained by Orchard [17], zero sensitivity of the filter transfer function to the variation of elements (in the passband) can be achieved if there are frequencies at which power transfer from the source to the load is maximal. The Chebyshev filter is also designed for maximum power transfer at certain frequencies (reflection zeroes) in the passband. Maximum power transfer for $n=9$ occurs at four frequencies where the transmission coefficient magnitude $\left|t_{n}(\mathrm{j} \omega)\right|^{2}$ equals

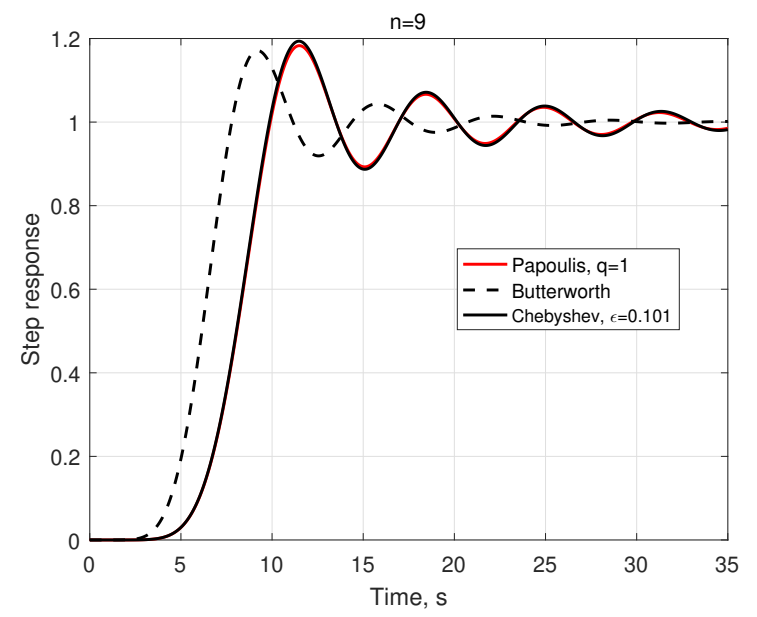

Fig. 5. Comparison of the step responses of equiripple and staircase filter.

one, and these values cannot be exceeded. At those frequencies Chebyshev filter realized as the LC ladder circuit exhibits zero sensitivity to variations in coil and capacitor values and keeps the sensitivity low through the whole passband [17]. As a consequence, with passband ripples decreasing, sensitivity also decreases. On the other hand, the Papoulis filter is not designed for the minimum passband sensitivity to element variations as the maximum power transfer occurs only at d.c. Therefore, the sensitivity in the passband of the Papoulis filter is much higher than sensitivity of its Chebyshev counterpart.

\section{Conclusion}

A new kind of allpole lowpass filters with the equiripple (Chebyshev) response in the passband has been proposed in this paper. A comparison of proposed with the allpole $\mathrm{Pa}$ poulis filters is discussed, because both filters have the same asymptotic slope. Table containing the Chebyshev transmission coefficients for filter degrees from 3 to 11 which are used for comparison with Papoulis filters, are presented. The main advantages of the proposed filters over the staircase filters can be listed as follows:

1. One additional degree of freedom allows to adjust the ripples in the pass-band, and thus the properties of the filter The counterpart of the staircase filter can be generated.

2. The Butterwort filter, as a special case of Chebyshev filter, is obtained for $\varepsilon \rightarrow 0$.

3. The characteristic function, reflection zeros, asymptotic and cutoff slope are expressed in a closed form.

4. The passband insertion loss and area under the characteristic function in the passband are smaller for every known staircase filter degree.

5. Numerical computation of the filter coefficients is simple. Coefficients of staircase filter are obtained by using complex mathematical apparatus. 
6. If the degree of filter increases then insertion loss and sensitivity to element changes decreases, while in the case of staircase filter the insertion loss and sensitivity increases.

7. The return loss is equiripple in the passband and its level is below the return-loss level of the Papoulis filters. The frequencies at which the return loss is zero are given in the mathematically closed form.

8. The passband sensitivity is zero at certain frequencies and it is significantly lower through the whole passband. At these frequencies, the filter characteristics can be further adjusted.

9. The odd degree characteristic function is perfectly square and it has zero at the origin, therefore, the realized LC ladder network is symmetric with an additional reduction of sensitivity which is not valid for the staircase filter.

Thus, it can be concluded that among filters with the staircase passband response and optimized cutoff slope and filters with the equiripple passband response, the latter offer a better solution in all applications. Since the design equations of the equiripple filter are simple and its performances are the same or better than the performances of the staircase filter, according to the comparison presented above, one gets the general feeling that the latter is mostly of academic importance.

\section{Acknowledgments}

The authors wish to thank Professor V. S. Stojanović of the University of Niš, Niš, Serbia for his valuable comments and suggestions. The work presented here was partly supported by the Serbian Ministry of Education and Science in the frame of the Projects TR 32009.

\section{References}

[1] PAPOULIS, A. Optimum filters with monotonic response. Proceedings of the IRE, 1958, vol. 46, no. 3, p. 906-609. DOI: 10.1109/JRPROC.1958.286876

[2] HALPERN, P. Optimum monotonic low-pass filters. IEEE Transactions on Circuit Theory, 1969, vol. 16, no. 2, p. 240-242. DOI: $10.1109 /$ TCT.1969.1082945

[3] RAKOVICH, B., LAZOVICH, S. Monotonic low-pass filters with improved stopband performance. IEEE Transactions on Circuit Theory, 1972, vol. 19, no. 2, p. 218-221. DOI: 10.1109/TCT.1972.1083420

[4] RAKOVICH, B., LITOVSKI, V. Monotonic passband low-pass filters with Chebyshev stopband attenuation. IEEE Transactions on Acoustics, Speech, and Signal Processing, 1974, vol. 22, no. 1, p. 39-44. DOI: 10.1109/TASSP.1974.1162538

[5] DJURICH, B., PETKOVICH, R. Generalized analysis of optimum monotonic low-pass filters. IEEE Transactions on Circuits and Systems, 1976, vol. 23, no. 11, p. 647-649. DOI: $10.1109 /$ TCS.1976.1084148
[6] BAEZ-LOPEZ, D., MINERO-MUNOZ, M., TINO-PARRA, M. A., et al. On the characteristics of monotonic L, Halpern, and parabolic filters. In Proceedings of the Midwest Symposium on Circuits and Systems. Covington (KY, USA), 2005, p. 1155-1158. DOI: 10.1109/MWSCAS.2005.1594311

[7] TOPISIROVIĆ, D., LITOVSKI, V., ANDREJEVIĆ STOŠOVIĆ, M. Unified theory and state-variable implementation of criticalmonotonic all-pole filters. International Journal of Circuit Theory and Applications, 2015, vol. 43, no. 4, p. 502-515. DOI: $10.1002 /$ cta.1956

[8] LITOVSKI, V. Electronic Filters: Theory, Numerical Recipes, and Design Practice based on the RM Software. Singapore Pte Ltd.: Springer Nature, 2019. ISBN: 9789813298521

[9] PERENIĆ, G., STAMENKOVIĆ, N. STOJANOVIĆ, N., et al. Chained-function filter synthesis based on the modified Jacobi polynomials. Radioengineering, 2018, vol. 27, no. 4, p. 1112-1118. DOI: $10.13164 /$ re.2018.1112

[10] ZVEREV, A. I. Handbook of Filter Synthesis. New York (USA): John Wiley \& Sons, Ltd., 1967. ISBN: 9780471749424

[11] STOJANOVIĆ, N., STAMENKOVIĆ, N., KRSTIĆ, I. Discretetime filter synthesis using product of Gegenbauer polynomials. Radioengineering, 2016, vol. 25, no. 3, p. 500-505. DOI: $10.13164 /$ re.2016.0500

[12] PAPOULIS, A. Optimum filters with monotonic response. Proceedings of the IRE, 1958, vol. 46, no. 3, p. 606-609. DOI: $10.1109 /$ JRPROC.1958.286876

[13] FUKADA, M. Optimum filters of even orders with monotonic response. IRE Transactions on Circuit Theory, 1959, vol. 6, no. 3, p. 277-281. DOI: 10.1109/TCT.1959.1086558

[14] BECCARY, C. The use of the shifted Jacobi polynomials in the sinthesis of lowpass filters. International Journal of Circuit Theory and Applications, 1979, vol. 7, no. 3, p. 289-295. DOI: $10.1002 /$ cta.4490070303

[15] STOJANOVIĆ, N., STAMENKOVIĆ, N., KRSTIĆ, I. Lowpass filters approximation based on modified Jacobi polynomials. Electronics Letters, 2017, vol. 53, no. 3, p. 140-142. DOI: 10.1049/el.2016.3025

[16] STOJANOVIĆ, N., STAMENKOVIĆ, N., ŽIVALJEVIĆ, D. Monotonic, critical monotonic, and nearly monotonic low-pass filters designed by using the parity relation for Jacobi polynomials. International Journal of Circuit Theory and Applications, 2017, vol. 45, no. 12, p. 1978-1992. DOI: 10.1002/cta.2375

[17] ORCHARD, H. J. Inductorless filters. Electronics Letters, 1966, vol. 2, no. 6, p. 224-225. DOI: 10.1049/el:19660190

\section{About the Authors...}

Negovan STAMENKOVIĆ was born in 1979. He received the M.Sc. degree from the Department of Electronics and Telecommunication at the Faculty of Technical Sciences, University of Priština, Kosovska Mitrovica in 2006, and the Ph.D. degree in Electrical and Computer Engineering from the Faculty of Electronic Engineering, Niš, Serbia, in 2011. $\mathrm{He}$ is professor at Faculty of Natural Sciences and Mathematics, University of Pristina. His current research interests lie in the area of analog and digital signal processing based on the residue number system. 
Nikola STOJANOVIĆ was born in 1973. He received his B.Sc. degree in Electronics and Telecommunication at the Faculty of Technical Sciences, University of Priština in 1997, M.Sc. degree in Multimedia Technologies at the Faculty of Electronic Engineering, University of Niš in 2013 and Ph.D. degree in Electrical and Computer Engineering from the Faculty of Electronic Engineering, Niš, Serbia, in 2018. Currently he works as a lecturer of multimedia and 3D animation at the Faculty of Electronics, University of Niš. His research interests are digital and analog signal processing, 3D animation and digitalization.

Dijana JOVANOVIĆ was born in 1996. She received the
B.Sc. and M.Sc. degrees from the Department of Informatics at the College of academic studies "Dositej", Belgrade, in 2018 and 2019. From 2018, she is working as a research assistant at the College of academic studies "Dositej". She is a Ph.D. student of Megatrend University of Belgrade, Serbia.

Željko STANKOVIĆ was born in 1957. He received the M.Sc. degree from the Department of Mathematics and Natural Sciences, University of Novi Sad, Serbia in 2006, and the Ph.D. degree in Information Technology from Department of Information Technolologies, University of Singidunum, Belgrade, Serbi, in 2010. He is assistant profesor at Faculty of Informatics, Paneuropean University Apeiron, Banja Luka. 